Swarthmore College

Works

8-1-2012

\title{
Sexual Trauma History Does Not Moderate Treatment Outcome In Attachment-Based Family Therapy (ABFT) For Adolescents With Suicide Ideation
}

\author{
G. Diamond \\ T. Creed \\ Jane Gillham \\ Swarthmore College, jgillha1@swarthmore.edu \\ R. Gallop \\ J. L. Hamilton
}

Follow this and additional works at: https://works.swarthmore.edu/fac-psychology

Part of the Psychology Commons

Let us know how access to these works benefits you

\section{Recommended Citation}

G. Diamond, T. Creed, Jane Gillham, R. Gallop, and J. L. Hamilton. (2012). "Sexual Trauma History Does Not Moderate Treatment Outcome In Attachment-Based Family Therapy (ABFT) For Adolescents With Suicide Ideation". Journal Of Family Psychology. Volume 26, Issue 4. 595-605. DOI: 10.1037/a0028414 https://works.swarthmore.edu/fac-psychology/82

This work is brought to you for free by Swarthmore College Libraries' Works. It has been accepted for inclusion in Psychology Faculty Works by an authorized administrator of Works. For more information, please contact myworks@swarthmore.edu. 


\title{
Sexual Trauma History Does not Moderate Treatment Outcome in Attachment-Based Family Therapy (ABFT) for Adolescents With Suicide Ideation
}

\author{
Guy Diamond \\ University of Pennsylvania and Children's Hospital of \\ Philadelphia, Philadelphia, Pennsylvania
}

\author{
Torrey Creed and Jane Gillham \\ University of Pennsylvania
}

Robert Gallop and Jessica Hamilton

Children's Hospital of Philadelphia, Philadelphia, Pennsylvania

\begin{abstract}
Despite the well-documented association between history of sexual trauma (HSA) and suicide ideation, HSA is largely overlooked in suicide treatment studies. Existing studies showed that patients with a HSA have a weaker treatment response. In this randomized clinical trial for suicide ideation, HSA did not moderate treatment outcome for Attachment-Based Family Therapy (ABFT). Adolescents responded better to ABFT than a control condition, regardless of HSA status. At baseline, adolescents with HSA were also more likely to report past suicide attempts than those without HSA, indicating that they are a particularly important subgroup to consider when developing and evaluating interventions that target suicide ideation. Findings suggest that ABFT is a robust intervention for suicide ideation regardless of HSA.
\end{abstract}

Keywords: adolescents, sexual abuse, suicide, depression, family therapy

Attachment Based Family Therapy (ABFT) is a manualized, empirically informed, family therapy model specifically designed to target family and individual processes associated with adolescent depression and suicide. ABFT emerges from interpersonal theories that suggest adolescent suicide can be precipitated, exacerbated, or buffered against by the quality of interpersonal relationships in families (Joiner \& Coyne, 1999). As a trust-based, emotion-focused psychotherapy, it aims to repair interpersonal ruptures and rebuild an emotionally protective, secure-based parent-child relationship (Diamond, Reis, Diamond, Siqueland, \& Isaacs, 2002; Diamond et al., 2010).

Several treatment studies have helped validate and refine this approach. An initial open trial of 10 cases demonstrated that ABFT resulted in significant decreases in adolescents' depression and suicidal ideation over 12 weeks (Diamond, Siqueland, \& Diamond, 2003). In a second study, 32 adolescents were randomized to ABFT or a 6-week wait list control (Diamond et al., 2002). Within the ABFT condition, $81 \%$ of patients no longer met criteria for Major Depressive Disorder (MDD) at posttreatment, compared to $47 \%$ of the waitlisted cases. In addition, $62 \%$ of subjects treated

Guy Diamond, Department of Psychiatry, School of Medicine, University of Pennsylvania, and Center for Family Intervention Science, Children's Hospital of Philadelphia, Philadelphia, Pennsylvania; Torrey Creed and Jane Gillham, Department of Psychology, University of Pennsylvania; Robert Gallop and Jessica Hamilton, Center for Family Intervention Science, Children's Hospital of Philadelphia, Philadelphia, Pennsylvania.

Correspondence concerning this article should be addressed to Guy S. Diamond, Center for Family Intervention Science, Children's Hospital of Philadelphia, 34th and Civic Center Boulevard, Philadelphia, PA 19104. E-mail: diamondg@email.chop.edu with ABFT met criteria for depression remission, compared to $19 \%$ of the control group. A third study randomized 66 adolescents to 12 weeks of ABFT or to Enhanced Usual Care (EUC) for reducing suicidal ideation and depressive symptoms (Diamond et al., 2010). Patients in ABFT demonstrated significantly greater rates of change on self-reported suicidal ideation at posttreatment and benefits were maintained at follow-up, with a strong overall effect size $(E S=.97)$. Treatment effects were also similar on clinician ratings. Significantly more patients in ABFT displayed clinical improvement on suicide ideation at posttreatment and follow up with a strong effect size $(\mathrm{OR}=4.41)$. Reduction in depression symptoms showed a similar pattern. These studies, several smaller pilot projects, and a host of treatment process studies, have earned ABFT the status of a "promising" empirically supported practice (David-Ferdon \& Kaslow, 2008; Promising Practices Network, 2011).

The growing evidence for the efficacy of ABFT gives merit to the investigation of treatment moderators. Treatment moderators help identify possible subgroups of a population who respond differentially to a treatment (Kazdin, 2001). Moderators may identify subpopulations with different causal mechanisms or courses of illness, thus helping to define which treatment is best suited for each patient. This type of investigation can prompt the strengthening, addition, or removal of intervention components to make the intervention more effective, efficient, or cost-efficient (Kraemer, Wilson, Fairburn, \& Agras, 2002).

Several factors indicate that the presence of a history of sexual abuse is an important moderator of treatment outcome among adolescents with suicide ideation. First, studies show a strong association between a history of sexual trauma (HSA) in childhood and presence of suicide ideation in adolescence or adulthood (Belik, Cox, Stein, Asmundson, \& Sareen, 2007). 
Adolescents with a HSA are more likely to have high levels of suicide ideation and attempts than those without a HSA (Beautrais, Joyce, \& Mulder, 1996). Adults with a childhood HSA are at greater risk for suicide ideation and behavior than adults without HSA, despite the temporal separation between childhood HSA and adult suicidality (Ullman \& Brecklin, 2002). Persons with a history of childhood trauma are also two to five times more likely to attempt suicide over their lifetime. This association between HSA and suicide is particularly ominous given that adolescents with a trauma history tend to be the most treatment resistant, especially for the treatment of depression and suicide (Oquendo et al., 2005). Better understanding of the impact of HSA in the treatment of suicidal adolescents might help improve treatments for this high risk population.

Second, several recent studies of depressed adolescents (a distinct but overlapping population with suicidal youth) found that adolescents with a HSA showed a weaker treatment response than those with no HSA, using predictor or moderation analyses. Using data from a study by Brent et al. (1997), Barbe, Bridge, Birmaher, Kolko, and Brent (2004) found that although cognitive-behavioral therapy (CBT) was more efficacious than Nondirective Supportive Therapy (NST), these differences washed out when patients had a history of HSA. Lewis et al. (2010) found that CBT in the Treatment of Adolescents with Depression Study (TADS, 2004) was also less effective when patients had a HSA, but not when patients had a history of physical abuse. Asarnow et al. (2009) found that those participants in the Treatment of SSRI-Resistant Depression in Adolescents (TORDIA; Brent et al., 2009) study with an abuse history (physical or sexual) responded poorly to combined treatment (CBT plus medication). These studies suggest that while CBT with and without medication remain best practice models for depressed adolescents, additional or alternative services may be needed when working with depressed adolescents with a HSA.

Surprisingly, no studies have explored the impact of a HSA on treatment for adolescents presenting with suicide ideation or attempts, a population very similar to these depressed patients. To address this question, we examined data from a recent ABFT trial targeting adolescents with severe suicide ideation and elevated depression symptoms (Diamond et al., 2010). The results of the overall study were promising, but the efficacy of ABFT among adolescents with a HSA is unknown and merits investigation. Therefore, the present study examined the following questions: Is a HSA associated with a more severe clinical presentation at study intake? Are the effects of ABFT (which has shown efficacy among adolescents with depression, suicide ideation and suicide behavior) moderated by a HSA?

\section{Method}

\section{Inclusions}

Adolescents were identified and initially screened for the study by staff in primary care and emergency rooms settings (see Diamond et al., 2010 for full methodical and demographic details). To be eligible, adolescents had to endorse 31 (i.e., clinical cut-off) or higher on the SIQ-JR (Reynolds, 1988) and above 20 (i.e., moderate depression) on the Beck Depression Inventory-II (BDI-II; Beck, Steer, \& Brown, 1996) on initial and second screening $(M=$ 2.21 days, $S D=1.34$ ), as evidence of sustained symptoms. For this high-risk group, a longer waiting period was deemed unethical. Among usual exclusion criteria (e.g., current psychosis), patients were ineligible if they had made a suicide attempt in the past 6 months or needed hospitalization. Patients could remain on antidepressant medication, if they had started it at least 12 weeks prior to randomization.

\section{Procedures}

We screened 341 adolescents over a 26 month period. Of those, 248 were not screened a second time because they either did not meet study criteria $(\mathrm{N}=188)$, were unavailable for the second screen $(\mathrm{N}=36)$, refused to participate $(\mathrm{N}=14)$, were hospitalized $(\mathrm{N}=6)$, or other reasons $(\mathrm{N}=4)$. Of the 93 who received the second screening, 11 no longer met study criteria, eight were unreachable prior to consent, and five refused study participation. Of the 69 who qualified at the second screening, three were diagnosed with psychotic features and thus excluded. In the end, 66 eligible adolescents and parents signed written informed consent and were randomized to ABFT or EUC. Assessments were conducted at baseline, 6 weeks (midtreatment), 12 weeks (posttreatment), and 24 weeks (follow-up). Of the final sample, 50 participants $(75 \%)$ were referred from primary care, and $16(25 \%)$ were referred from emergency departments. The study was approved by the IRB at The Children's Hospital of Philadelphia. As part of consent, patients gave permission for clinical material to be used for educational purposes.

\section{Participants}

In the final sample, the mean age was 15.1 years $(S D=1.5), 49$ (74\%) were African American, and 55 (83\%) were female. Of the parents, $27(41 \%)$ had an income under \$30,000, 17 (26\%) were married, and $46(70 \%)$ had no more than a high school diploma. None of the participating parents were the perpetrator of the sexual abuse. Diagnostically, 31 (47\%) adolescents met criteria for a depressive disorder, $44(67 \%)$ met criteria for an anxiety disorder, and $38(57 \%)$ met criteria for an externalizing disorder (i.e., ADHD, oppositional, or conduct disorder). Forty-one participants $(62 \%)$ reported a past suicide attempt, and nearly three quarters (73\%) of these participants (30 of the 41 attempters) reported multiple attempts. Upon study entry, six patients were taking an antidepressant medication, three in each treatment condition. There were no statistical differences between treatment groups on demographic and clinical variables.

\section{Defining History of Sexual Trauma}

Adolescents with a HSA were identified based upon several criteria: (a) demographic form ["Were you physically or sexually abused?" $(\mathrm{N}=5)]$; (b) the Reasons for Suicide measure (Diamond \& Wintersteen, 2007) ["Were you forced to do something sexual (Yes/No)" (N = 7)]; (c) the DISC-IV (Shaffer, Fisher, \& Lucas, 1997) Post-Traumatic Stress Disorder (PTSD) section (adolescent) ["Have you ever been very upset by someone forcing you to do something sexual that you really didn't want to do?" $(\mathrm{N}=21)$ or "Have you ever been attacked sexually or raped?" $(\mathrm{N}=13)]$; and (d) DISC-IV PTSD section as reported by the parent. We use the term "history of sexual trauma (HSA)" because positive endorse- 
ment of the items may include rape, molestation, or other forms of sexual victimization.

Accounting for overlap in indicators, 30 adolescents, or $45 \%$ (ABFT, $\mathrm{N}=19$; EUC, $\mathrm{N}=11$ ) of the total sample reported a HSA. Ten adolescents reported trauma that was not endorsed by parents, and nine parents reported trauma not endorsed by the adolescent. Given the varied nature of the trauma history being reported, this level of inconsistency was expected. All adolescents who answered "yes" to the Demographic question (a) and Reasons for Suicide question (b) also answered "yes" to item (c) in the DISC. When sexual abuse was indicated, this information was brought to the attention of the project coordinator, a licensed psychologist and discussed with the family. If the family was assigned to ABFT, this information was given to the therapist to discuss in the treatment. If the patient was assigned to EUC, the project coordinator discussed this directly with the patient and the parents in order to assess the need for mandatory reporting. One case was reported during the therapy. All other cases had been reported or reporting was not deemed necessary.

\section{Treatments}

ABFT. Based on this theoretical foundation, the first half of treatment focuses on helping the adolescent identify and discuss past and present family conflicts that have strained the attachment bond and damaged trust. This includes targeting how these relational failures contribute to the suicidal thinking or how they inhibited parents from providing safety and protection (e.g., going to parents when feeling suicidal). The second half of treatment focuses on promoting adolescent autonomy (i.e., improving school productivity, finding a job, developing or returning to social activities, etc.). This strategy decreases isolation and increases the adolescent's positive experiences.

Treatment is organized around five treatment tasks, each of which might take one to three sessions. A Relational Reframe Task aimed to reduce criticism and hostility. The pivotal question in this task is: "Why don't you go to your parents when you are feeling suicidal?" This question shifts the focus of therapy from the fixing the adolescent to repairing the relational ruptures. The Alliance Building Task with the Adolescent focuses on strengthening treatment engagement and preparing for the upcoming attachment task. The session focuses on strengthening alliance, identifying core family dynamics that fuel conflict, and encouraging the adolescent to discuss these issues with a parent in the course of treatment. The Alliance Building Task with the Parent(s) focuses on reducing parental distress and improving parenting practices. This begins with understanding parents' current stressors (e.g., psychiatric distress, marital problems), and then explores parents' own childhood experiences of attachment ruptures. As parents remember their own longing for attachment security, they became more motivated to provide it to their children, and thus, more willing to learn emotion focused parenting skills (à la Gottman, Katz, \& Hooven, 1996).

The Reattachment Task culminates the work of the previous sessions. The session begins with the adolescent disclosing past and present family events or dynamics that violate(d) the attachment bond and damage(d) trust. As parents respond empathetically, adolescents are more forthcoming (Diamond \& Liddle, 1999). Parents often acknowledge, if not apologize, for past at- tachment failures, which promotes forgiveness and renewed mutual trust, thus revising adolescent expectancy and desire for parents protection and support. Most importantly, this task diffuses AQ: $\mathbf{5}$ family tension and increases the likelihood that an adolescent would go to his parent the next time he feels suicidal.

The Competency Promoting Task focuses on building adolescent self-esteem through promoting autonomy (e.g., improving academics, finding a job, developing social activities). Experiences of competency can buffer against further hopelessness and depression, thus reducing thoughts of suicide. With trust and attachment Fn6-7 on the mend, the family serves as a secure base from which an adolescent can explore these goals, and learn problem-solving skills, affect regulation and perspective taking.

Enhanced Usual Care (EUC). EUC was a facilitated referral process with ongoing clinical monitoring. Research staff found mental health care providers in the local community, set up initial appointments, and encouraged patient attendance. The treating therapist was responsible for engaging and retaining adolescents in treatment. As reported in Diamond et al. (2010), EUC patients received treatment at community mental health centers $(77 \%)$ or at an independent practice $(23 \%)$. The majority $(52 \%)$ received individual therapy, while $19 \%$ participated in group therapy, $6 \%$ received family therapy, $3 \%$ received case management. Nineteen percent $(19 \%)$ did not attend treatment. There were no differences in the completion rates of assessments between the two treatment groups at any time points.

\section{Outcome Measures}

Suicide Ideation Questionnaire-JR (SIQ-JR; Reynolds, 1988). The SIQ-JR is a 15 -item version of the SIQ. The SIQ-JR is scored on a continuum $(0=$ Inever had this thought, $6=$ Almost every day) within the last month. A score of 31 or above and six critical items (e.g., "I have a plan to kill myself") indicate serious suicide risk. Test-retest reliability was .89 over 3 weeks (Reynolds \& Mazza, 1999). In our sample, internal consistency was Cronbach's alpha $=.85-0.94$. In this study, clinical recovery on the SIQ-JR was defined as a score $\leq 13$, based on normative data from an African American inner city sample (Reynolds \& Mazza, 1999).

Scale for Suicidal Ideation-Past Week (SSI-PW; Beck et al., 1979). The SSI-PW is a 19-item interviewer-administered AQ: 8 rating scale measuring suicidal ideation in the past week. Each item is rated on a $0-3$ Likert scale. Scores are summed to get a total score ranging from $0-38$. Patients are asked about wanting to die, desire to make an active or passive suicide attempt, duration and frequency of ideation, sense of control over making an attempt, number of deterrents, and amount of actual preparation for a contemplated attempt. The SSI-PW is strongly correlated with suicide items from the BDI and the HAM-D (Beck et al., 1979). The SSI is most notable for its predictive validity; patients who score in the higher risk category were 14 times more likely to commit suicide than patients who scored low (Beck et al., 1979). AQ: 10 In the current sample, the measure had high internal consistency (Cronbach's alpha $=.82-.97)$.

Beck Depression Inventory-II (BDI-II; Beck et al., 1996). The BDI-II is a 21-item self-report instrument designed to assess the severity of depressive symptoms in adults and adolescents. Item scores range from 0 to 3 , with the total ranging from $0-63$. 
The BDI-II is highly and positively correlated with other measures of depression and has good sensitivity and specificity. In a study with African American patients, reliability was demonstrated with high internal consistency (.90), good item-total intercorrelations, and good criterion-related validity (Grothe et al., 2005). The original BDI was significantly correlated with the subscales of Withdrawn $(r=35, p=.04)$ and Anxiety $(r=.46, p=.008)$ on the AQ: 11 Youth Self-Report (Diamond et al., 2002). The BDI-II had excellent internal consistency (Cronbach's alpha $=.86-.95$ ) in the current sample.

The Self-Report of Family Functioning (SRFF). The SRFF is composed of 15, 5-item factors (e.g., democratic family style) with Cronbach's alpha from .63-.91, with most in the .70-.85 range. Analyses have yielded highly reliable and stable factors. For this article, we only used the conflict and cohesion scales.

Diagnostic Interview Schedule for Children (Present-State Voice; V-DISC; Shaffer et al., 1997). This is a computer administered version of this widely used diagnostic tool for research with children and adolescents.

\section{Data Analysis}

Inspection, transformation, and analytic framework of the data. Our modeling framework is based on extension of the General Linear Model (GLM) that assumes normally distributed data. This assumption was tested for each outcome. Box-Cox transformations indicated a square-root transformation would correct the deviations in normality for the positively skewed SIQ-JR and BDI-II. Transformation did not substantially improve normality for the SSI, which ranged from $0-7$, but postbaseline transformation consisted of a high prevalence $(37.5 \%-64.8 \%)$ of 0 scores at each assessment. Therefore, following common convention (Brown et al., 2005) the scores were dichotomized. Zero was coded as "no current suicide ideation" (clinical recovery) and scores above 0 were coded as "current suicide ideation." We used pattern-mixture models to examine whether missing data were related to change in outcome over time (Hedeker \& Gibbons, 1997).
Statistical power. Power calculations of a significant HSA $x$ treatment interaction were implemented through SAS power procedure PROC GLMPOWER. To estimate an expected effect size, we used the contrast of Placebo (PBO) and CBT at endpoint found in the TADS study (Lewis et al., 2010). In the TADS study, trauma moderated treatment outcome with an approximate effect size of 0.3 for the CBT-PBO comparison. CBT did well with the nontrauma group but not with the patients reporting a history of trauma. In the sexual abuse subgroup, CBT was associated with worse outcomes than PBO with an effect size of -1.0. Effect size calculations are based on the difference in the adjusted CDRS-R total score and pooled baseline standard deviation (see Lewis et al., 2010, Figures 1.1 to 1.4 and Table 1). To replicate these TADS F1,T1 findings, our study would have $71.5 \%$ power to detect this interaction as statistically significant at the 0.05 level. If the effects were slightly larger, $(d=0.4$ in the nontrauma group and $d=$ -1.1 in the sexual trauma group) then the study would have $83.1 \%$ power to detect a statistically significant interaction at the 0.05 level. For contrast within the HSA group, the study would have $82.4 \%$ power to yield a statistically significant superiority result, thus replicating the findings of Lewis et al. (2010) for the EUC and ABFT contrast within the HSA group.

\section{Results}

\section{Question 1: Do Adolescents with a HSA Have a More Severe Clinical Presentation at Baseline Than Adolescents Without a HSA?}

We used $t$ tests for continuous measures, chi square analyses for categorical measures, and Fisher's exact tests for categorical measures with cells of 5 or less. Adolescents with or without a HSA were similar across most demographic variables (see Table 1), although adolescents with a HSA were almost exclusively females (96.7\%). This finding is consistent with the overall higher prevalence rates of a HSA among females (Center for Disease Control, 2005). Adolescents with a HSA were also almost twice as likely to

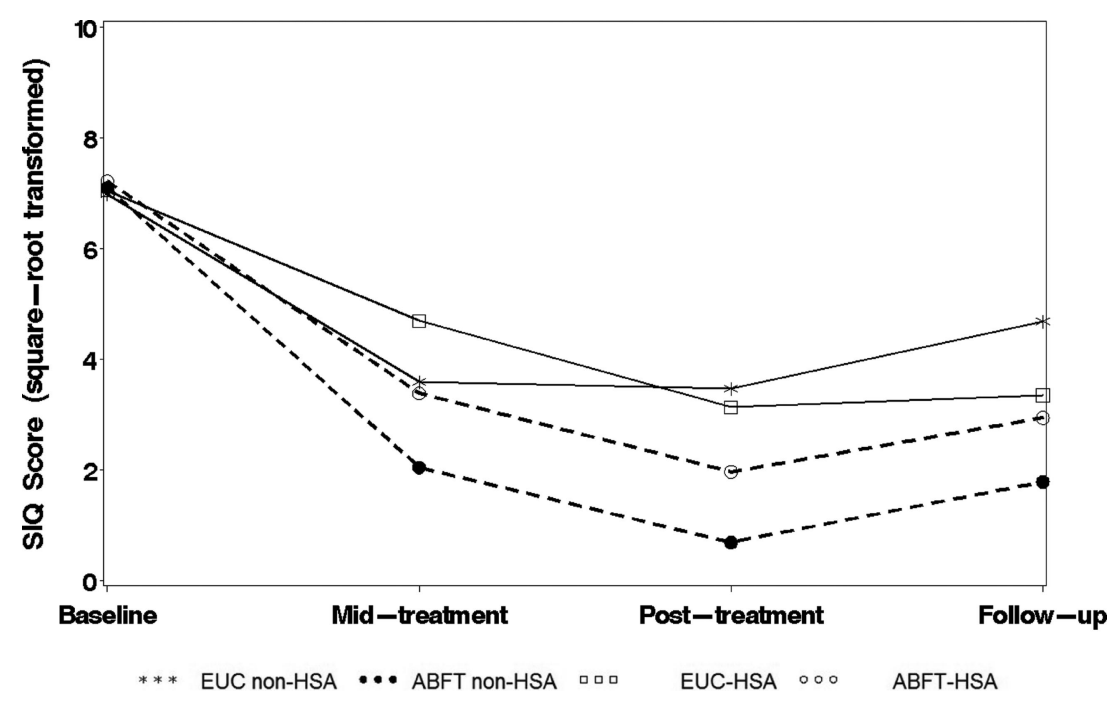

Figure 1. Rate of change in ABFT and EUC on SIQ-JR. 
Table 1

Baseline Characteristics of Adolescents With and Without an HSA

\begin{tabular}{lcccc}
\hline & $\begin{array}{c}\text { Sexual trauma } \\
(n=30)\end{array}$ & $\begin{array}{c}\text { No sexual trauma } \\
(n=36)\end{array}$ & Statistic, $d f$ & $p$ value \\
\hline Demographic Characteristics & & & & \\
Age in years, Mean (SD) & $15.43(1.5)$ & $15.00(1.7)$ & $t=-1.10,64$ & 0.28 \\
African American race, No. (\%) & $20(66.7 \%)$ & $29(80.6 \%)$ & $\chi=1.65,1$ & 0.20 \\
Female sex, No. (\%) & $29(96.7)$ & $26(72.2)$ & $\chi=7.04,1$ & 0.01 \\
Income, less than 30,000, No. (\%) & $14(50.0)$ & $13(39.4)$ & $\chi=.69,1$ & 0.41 \\
Single parent household, No. (\%) & $23(79.3)$ & $24(68.6)$ & $\chi=.94,1$ & 0.33 \\
Social Services Involvement, No. (\%) & $16(53.3)$ & $9(25.7)$ & $5.21,1$ & 0.02 \\
Family Measures & & & & \\
Family functioning- Conflict & $11.97(3.39)$ & $12.47(3.27)$ & $t=.62,64$ & 0.54 \\
Family functioning- Cohesion & $13.73(3.50)$ & $14.14(3.59)$ & $t=.46,64$ & 0.65 \\
Family history of suicide attempt, No. (\%) & $8(27.6)$ & $12(36.4)$ & $\chi=.54,1$ & 0.46 \\
Parent sexual abuse, No. (\%) & $6(20.0)$ & $7(19.4)$ & $\chi=.003,1$ & 0.96 \\
Clinical Characteristics, Mean (SD) & & & & \\
SIQ-JR & $51.8(11.85)$ & $50.47(14.73)$ & $t=-.40,64$ & 0.69 \\
Beck's Depression Inventory-II & $34.3(8.07)$ & $31.89(9.7)$ & $t=-1.08,64$ & 0.28 \\
Scale for Suicide Ideation & $9.38(6.24)$ & $8.12(8.62)$ & $t=-.66,61$ & 0.52 \\
Current Psychiatric Diagnoses, No. (\%) & & & & \\
Major depressive episode-Past year & $17(48.6)$ & $18(51.4)$ & $\chi=0.30,1$ & 0.59 \\
Any anxiety & $27(90.0)$ & $\mathbf{2 5 ( 6 9 . 4 )}$ & $=\mathbf{4 . 1 4 , 1}$ & 0.04 \\
Oppositional and/or conduct & $16(53.3)$ & $19(52.8)$ & $\chi=.002,1$ & 0.96 \\
Substance abuse and/or dependence & $6(20)$ & $8(22.2)$ & $\chi=.05,1$ & 0.83 \\
Clinical History, No. (\%) & & & \\
Adolescent attempted suicide & $25(83.3)$ & $15(41.7)$ & $\chi=11.90,1$ & 0.001 \\
Multiple attempts & $21(70)$ & $9(25)$ & $\chi=13.37$ & 0.001 \\
Adolescent past psych. Hospitalization & $7(24.1)$ & $7(20.0)$ & $\chi=.16,1$ & 0.69 \\
\hline
\end{tabular}

have a history of contact with the Department of Human Services, likely reflecting a report of the HSA. The groups were similar in reported psychopathology (see Table 1), although adolescents with a HSA were almost twice as likely to report a past suicide attempt and three times more likely to report multiple attempts. These significant predictors were used to create a propensity score for each individual to correct for baseline imbalances in the subsequent analyses.

\section{Question 2: Are Treatment Effects Moderated by a HSA?}

Suicide measures. Hierarchical linear models (HLM) for continuous outcomes, which accommodate the clustering due to the repeated assessments of individuals, were used to examine the moderation effect of HSA on the intervention effect. For the binary outcomes, including clinical response (SSI) and remission (SIQJR, BDI), we implemented a hierarchical generalized linear model (HGLM). HLM models individual change as a function of time such as linear, quadratic, cubic, and piecewise. Preliminary HLM structures varied dependent on the treatment of time, where we consider change over time as follows: linear, quadratic, cubic, and the piecewise model described in Diamond et al. (2010). We compared model fit statistics [ -2 Log-likelihood functions, the Akaike information criterion (AIC), and the Akaike corrected information criterion (AICC)] to determine the best structure. We included trauma history (HSA vs. non-HSA) intervention condition, and all respective interactions with change over time. An interaction between trauma history and change over time would indicate differential change dependent on trauma history. Similarly, an interaction of trauma history with intervention condition and change over time would indicate differential intervention effects dependent on trauma history (i.e., that intervention effects were moderated by HSA).

Based on our previous finding of baseline differences between HSA and non-HSA adolescents in question 1, we used propensity scores to disentangle the potential confound of baseline discriminators of trauma history (Rosenbaum, 2002; Gelman \& Hill, 2007). Based on a logistic regression framework, predicted probabilities of having a HSA (propensity scores) were estimated for each individual, which provided a summary of the covariate imbalance across HSA/non-HSA groups. The distributions of propensity scores across the two groups are usually different, with some regions of nonoverlap in the tails. The sample was too small $(\mathrm{N}=66)$ to match participants across groups using propensity scores (Rosenbaum, 2002). Therefore, propensity scores were included as a covariate in the moderation analyses, to adjust for significant baseline discriminators.

For both measures of suicide ideation (SIQ-JR, SSI), the best fitting HLM model was determined to be piecewise, modeling the rate of change from baseline to 12 weeks (end of active treatment) and then again at 24 weeks (follow-up). Comparisons of slope estimates from baseline to posttreatment suggest that ABFT was more effective than EUC in reducing suicide ideation, regardless of adolescents' HSA [SIQ-JR: $F(1,61)=11.25, p=.01$; SSI: $F(1$, $61)=4.87, p=.03]$. These benefits were maintained at follow up. Therefore, HSA did not significantly moderate ABFT's effect on suicide ideation. HLM analyses predicting SIQ-JR and SSI scores revealed no significant interactions between trauma history and intervention condition in rates of change over time [SIQ-JR baseline to posttreatment: $F(1,61)=1.67, p=.20$; SIQ-JR posttreat- 
ment to follow-up: $F(1,61)=0.30, p=.59$; SSI, baseline to posttreatment: $F(1,61)=0.67, p=.41$; posttreatment to follow-up: $F(1,61)=1.83, p=.18$ ]. Table 2 shows rates of change and Figures 1-3 show outcome scores by HSA and intervention condition.

For depression (BDI-II), the best fitting HLM structure was a piecewise model run first from baseline to 6 weeks (midtreatment) and then to 24 weeks (follow-up). Comparisons of slope estimates suggest ABFT resulted in better outcomes than EUC from baseline to midtreatment regardless of HSA status, $F(1,61)=5.89, p=$ .017. Like the main findings, there were no differential outcomes at posttreatment and follow-up, but the benefits gained at posttreatment were retained at follow-up. HSA did not significantly moderate ABFT's effect on depression. HLM analyses predicting BDI-II scores revealed no significant interactions between trauma history and intervention condition in rates of change over time [BDI baseline to midtreatment: $F(1,61)=0.42, p=.54$; BDI midtreatment to follow-up: $F(1,61)=2.72, p=.10]$. ABFT resulted in better outcomes, compared to EUC, within both HSA and non-HSA adolescents, although these pairwise differences were not statistically significant. Unlike the SSI and SIQ-JR, depressive symptoms declined more for non-HSA than HSA adolescents in EUC, but equally among HSA and non-HSA adolescents in ABFT during the early portion of change (from baseline to midtreatment).

Recovery rates. We used Fisher's exact tests (due to reduced sample size) to examine whether ABFT led to greater clinical response than EUC among participants with an HSA. A comparison of the posttreatment recovery rates (see Table 2 for both SIQ-JR and SSI) suggested that ABFT was superior to EUC regardless of trauma history (SIQ-JR: $\chi^{2}(1, \mathrm{~N}=60)=8.93, p=$ .003 ; SSI: $\left.\chi^{2}(1, \mathrm{~N}=52)=6.24, p=.013\right)$. HSA also did not moderate remission in treatment. Logistic regression analyses predicting clinical response on SIQ-JR, SSI, and BDI revealed no significant interactions between trauma history and intervention condition, SIQ-JR: $\chi^{2}(1, \mathrm{~N}=60)=0.01, p=.99 ; \mathrm{SSI}: \chi^{2}(1, \mathrm{~N}=$ $52)=1.85, p=.17$, BDI: $\chi^{2}(1, \mathrm{~N}=60)=1.01, p=.32$. (Figures 4 and 5 shows remission rates for the SIQ-JR and BDI-II, which F4,F5 was similar for the SSI.)

Examination within ABFT. Statistical contrasts within the HLM models above examined if, within ABFT, the rates of change for the respective outcomes were significantly different for adolescents with and without a HSA. For all outcomes we found nonsignificant effects [SIQ-JR baseline to posttreatment: $t(61)=-1.15, p=.26$; SIQ-JR posttreatment to follow-up: $t(61)=-0.17, p=.87$; SSI, baseline to posttreatment: $t(61)=$ $1.14, p=.26$; posttreatment to follow-up: $t(61)=-1.00, p=$ .32; BDI, baseline to midtreatment: $t(61)=-0.66, p=.51$; midtreatment to follow-up: $t(61)=-1.46, p=.15]$. No statistical significance was found suggesting there were no statistical differences between the HSA and non-HSA within ABFT.

As discussed, the lack of statistical significance does not mean we can infer that ABFT is equally effective for those with and without a HSA. To answer this question of whether ABFT is equally effective for patients with and without a HSA would require formal Bioequivalence testing and a very large sample size. Therefore, to quantify the "similarity" in the presence of a nonsignificant contrast, we derived the effect size for the differences between the two groups. Effect size was measured using Cohen's $d$ for the continuous measures and odds ratios (OR) for binary

Table 2

Estimated Rates of Change and Estimated Outcomes by HSA and Intervention Status

\begin{tabular}{|c|c|c|c|c|c|c|}
\hline \multirow[b]{2}{*}{ Outcome } & \multicolumn{2}{|c|}{ No sexual trauma } & \multicolumn{2}{|c|}{ Sexual trauma } & \multicolumn{2}{|c|}{ Sexual trauma: ABFT versus EUC } \\
\hline & $\begin{array}{c}\text { ABFT } \\
(n=16)\end{array}$ & $\begin{array}{c}\text { EUC } \\
(n=20)\end{array}$ & $\begin{array}{c}\text { ABFT } \\
(n=19)\end{array}$ & $\begin{array}{c}\text { EUC } \\
(n=11)\end{array}$ & Effect size & Statistic \\
\hline \multicolumn{7}{|l|}{ SIQ-JR } \\
\hline Early slope (est $\pm s e)$ & $-1.93 \pm 0.19$ & $-1.03 \pm 0.17$ & $-1.66 \pm 0.22$ & $-1.27 \pm 0.22$ & $d=0.73$ & $F(1,29)=4.05, p=.05$ \\
\hline Late slope $(\mathrm{est} \pm s e)$ & $0.16 \pm 0.09$ & $0.20 \pm 0.08$ & $0.16 \pm 0.09$ & $0.09 \pm 0.12$ & $d=0.30$ & $F(1,29)=0.69, p=.41$ \\
\hline Posttreatment $(\mathrm{mn} \pm s d)$ & $1.86 \pm 3.48$ & $17.67 \pm 3.07$ & $7.94 \pm 3.16$ & $13.73 \pm 3.83$ & $d=0.44$ & $F(1,29)=1.77, p=.19$ \\
\hline Follow-up $(\mathrm{mn} \pm s d)$ & $6.07 \pm 4.08$ & $26.22 \pm 3.60$ & $14.13 \pm 3.82$ & $15.75 \pm 5.05$ & $d=0.15$ & $F(1,29)=0.16, p=.69$ \\
\hline Midtreatment Remission rate & $80.0 \%$ & $50.0 \%$ & $61.1 \%$ & $27.3 \%$ & $\mathrm{OR}=4.18$ & $\chi^{2}(1)=3.13, p=.08$ \\
\hline Posttreatment Remission rate & $92.9 \%$ & $61.1 \%$ & $82.4 \%$ & $36.4 \%$ & $\mathrm{OR}=8.11$ & $\chi^{2}(1)=6.15, p=.02$ \\
\hline Follow-up Remission rate & $78.6 \%$ & $33.3 \%$ & $62.5 \%$ & $37.5 \%$ & $\mathrm{OR}=3.48$ & $\chi^{2}(1)=1.34, p=.25$ \\
\hline \multicolumn{7}{|r|}{ 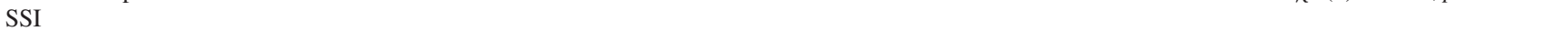 } \\
\hline Early slope (est $\pm s e)$ & $-0.62 \pm 0.22$ & $-0.26 \pm 0.19$ & $-1.10 \pm 0.26$ & $-0.31 \pm 0.35$ & $d=0.66$ & $F(1,29)=3.27, p=.08$ \\
\hline Late Slope (est $\pm s e$ ) & $-0.19 \pm 0.14$ & $0.002 \pm 0.10$ & $-0.05 \pm 0.13$ & $-0.22 \pm 0.16$ & $d=0.30$ & $F(1,29)=0.69, p=.41$ \\
\hline Midtreatment Remission rate & $50.0 \%$ & $31.2 \%$ & $33.3 \%$ & $37.5 \%$ & $\mathrm{O} R=.80$ & $\chi^{2}(1)=0.04, p=.84$ \\
\hline Posttreatment Remission rate & $69.2 \%$ & $47.1 \%$ & $69.2 \%$ & $11.1 \%$ & $\mathrm{OR}=17.99$ & $\chi^{2}(1)=7.25, p=.007$ \\
\hline Follow-up Remission rate & $85.7 \%$ & $44.4 \%$ & $78.6 \%$ & $50.0 \%$ & $\mathrm{OR}=3.67$ & $\chi^{2}(1)=1.92, p=.17$ \\
\hline \multicolumn{7}{|l|}{ BDI-II } \\
\hline Early slope (est $\pm s e)$ & $-1.68 \pm 0.25$ & $-1.30 \pm 0.24$ & $-1.16 \pm 0.24$ & $-0.36 \pm 0.30$ & $d=1.00$ & $F(1,29)=7.56, p=.007$ \\
\hline Late slope (est $\pm s e)$ & $0.04 \pm 0.07$ & $-0.11 \pm 0.07$ & $-0.13 \pm 0.07$ & $-0.03 \pm 0.09$ & $d=0.30$ & $F(1,29)=0.70, p=.41$ \\
\hline Posttreatment $(\mathrm{mn} \pm s d)$ & $9.43 \pm 3.42$ & $13.33 \pm 3.02$ & $15.18 \pm 3.16$ & $26.90 \pm 3.86$ & $d=1.16$ & $F(1,29)=10.17, p=.002$ \\
\hline Follow-up $(\mathrm{mn} \pm s d)$ & $11.92 \pm 3.29$ & $10.94 \pm 2.90$ & $12.71 \pm 2.99$ & $28.00 \pm 4.36$ & $d=0.85$ & $F(1,29)=5.47, p=.02$ \\
\hline Midtreatment Remission rate & $53.3 \%$ & $18.8 \%$ & $17.7 \%$ & $0 \%$ & $\mathrm{OR}=\mathrm{N} \cdot \mathrm{D}$ & Fisher's exact $p=.26$ \\
\hline Posttreatment Remission rate & $64.3 \%$ & $44.4 \%$ & $47.1 \%$ & $9.1 \%$ & $\mathrm{OR}=8.99$ & Fisher's exact $p=.049$ \\
\hline Follow-up Remission rate & $50.0 \%$ & $50.0 \%$ & $64.7 \%$ & $12.5 \%$ & $\mathrm{OR}=12.83$ & Fisher's exact $p=.03$ \\
\hline
\end{tabular}

Note. Values for slope and estimated outcomes represent the estimate \pm standard error. N.D. indicates odds ratio is not defined. 


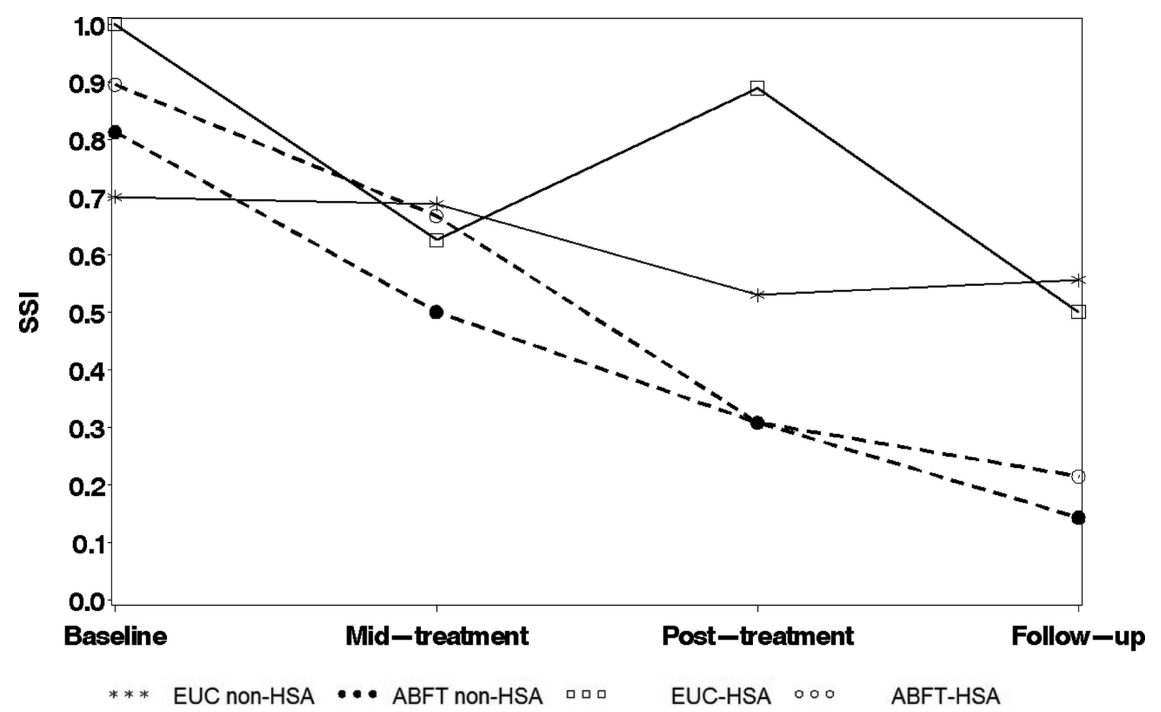

Figure 2. Rate of change in ABFT and EUC on SSI.

measures (i.e., response and remission). The OR corresponded to, on average, the number of times an event was more likely to occur for ABFT compared to EUC at each assessment. For Cohen's $d$, positive effects sizes reflect benefits of ABFT relative to EUC.

To quantify the difference, we derived effects sizes for each contrast with propensity scores per subject included as a covariate [SIQ-JR baseline to posttreatment: $d=+0.29$; SIQ-JR posttreatment to follow-up: $d=-0.04$; SSI, baseline to posttreatment: $d=$ -0.29 ; posttreatment to follow-up: $d=+0.25$; BDI, baseline to midtreatment: $d=+0.17$; midtreatment to follow-up: $d=-0.37$ ] All effect sizes of the contrast fell below the medium effect size threshold, suggesting that minimal differences were found between the outcomes in ABFT, with the adjusted covariate imbalance. Positive effect sizes indicate non HSA subjects had the best improvement and negative effect sizes indicate the HSA subjects had best improvement. Still, the small effect sizes indicate minimal differences between the outcomes of the two groups within ABFT.

\section{Question 3: Is ABFT Superior to EUC Among Adolescents With a HSA $(\mathbf{N}=30)$ ?}

Continuous measures. The rate of reduction in the transformed SIQ-JR scores was significantly greater within ABFT/HSA compared to EUC/HSA from baseline to posttreatment: $F(1,29)=$ $4.05, p=.05, d=0.73$. From posttreatment to follow-up, there was no difference in rate of improvement between ABFT/HSA and EUC/HSA, $F(1,29)=0.69, p=.41, d=.30$, although gains made in ABFT during treatment were maintained at posttreatment and follow-up. For SSI, there were no significant treatment effects from baseline to posttreatment, posttreatment to follow-up, or on

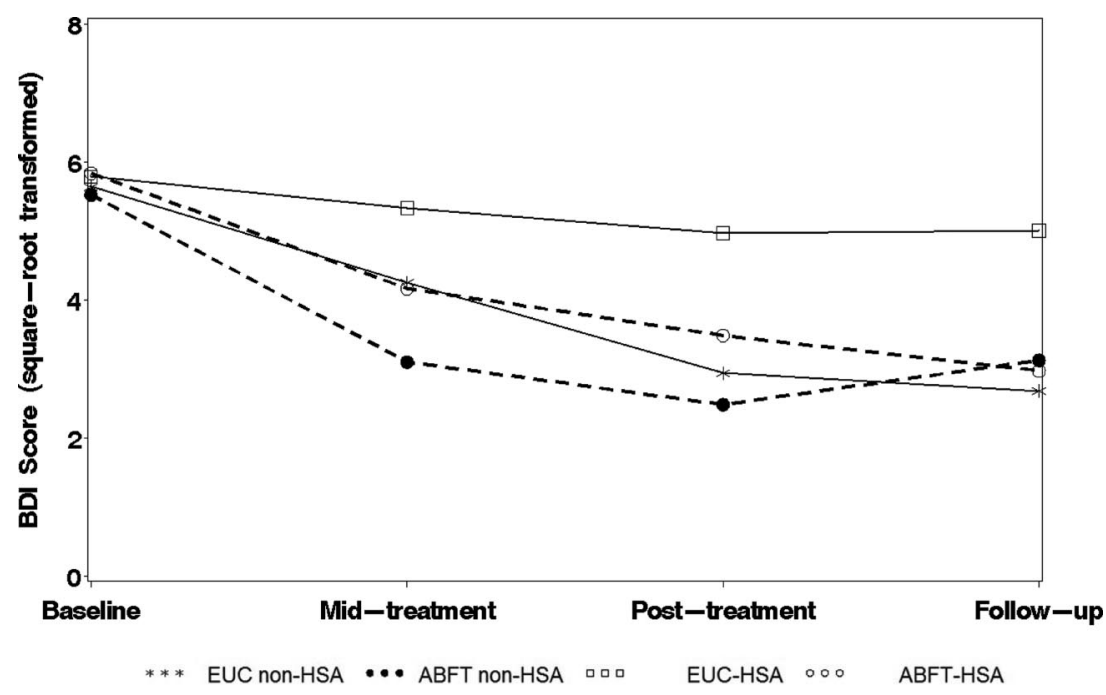

Figure 3. Rate of change in ABFT and EUC on BDI-II. 


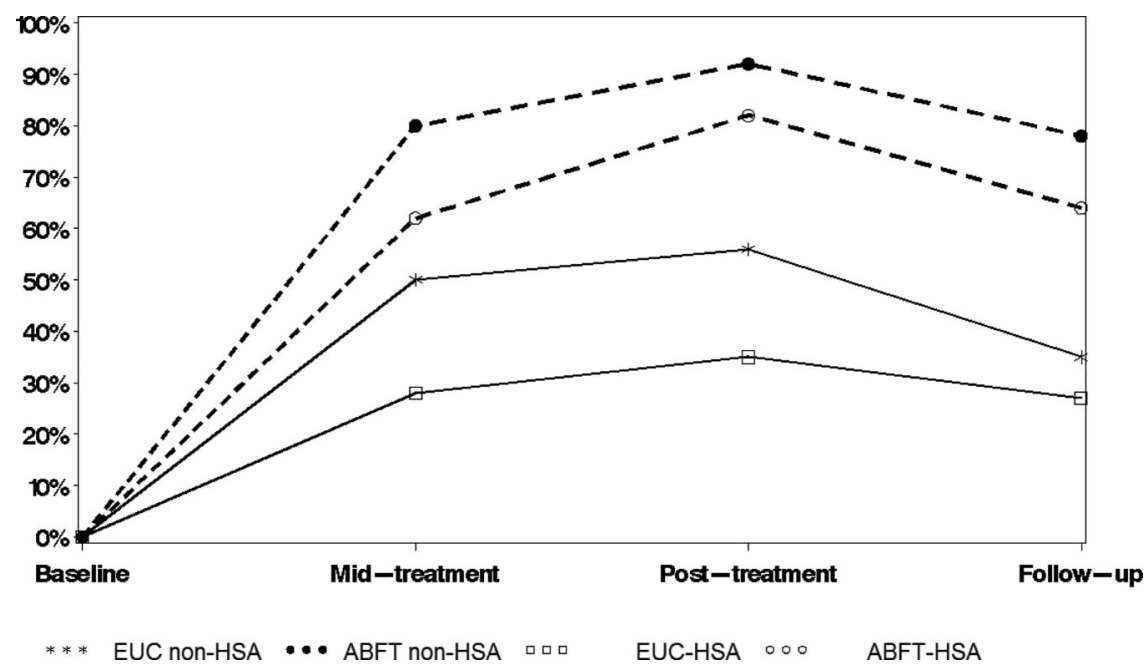

Figure 4. Proportion scoring in normative range on suicide ideation (SIQ).

total change. The rate of reduction in depression scores (BDI) was greater within ABFT/HSA compared to EUC/HSA from baseline to midtreatment: $F(1,29)=7.56, p=.007, d=1.00$. However, from midtreatment to follow-up, there was no difference in rate of improvement between ABFT and EUC, $F(1,29)=0.70, p=.41$, $d=.30$, although gains made in ABFT during treatment were maintained. Except for the SSI findings, these results closely parallel the main findings (Diamond et al., 2010). In addition, the Lewis et al. (2010) study found an effect size of $d=0.81$ in the contrast of CBT versus PBO among its sexual abuse group during the studies treatment period, in favor of PBO. We found a similar magnitude of effect $(d=0.73)$ for the SIQ-JR and a $d=1.00$ for BDI, but the results are in favor of ABFT.

Remission rates. Among adolescents with a HSA, remission rates on the SIQ, SSI and BDI-II were higher in ABFT than EUC (see Table 2, columns 4-7). These differences were significant at several assessment points. On the SIQ, remission rates were $61.1 \%$ for ABFT versus $27.3 \%$ for EUC, $\chi^{2}(1)=3.13, p=.08$, OR $=$
4.18. Posttreatment remission rates were $82.4 \%$ for ABFT versus $36.4 \%$ for EUC, $\chi^{2}(1)=6.15, p=.02$, OR $=8.11$. Follow-up remission rates were $62.5 \%$ for ABFT versus $37.5 \%$ for EUC, $\chi^{2}(1)=1.34, p=.25, \mathrm{OR}=3.48$. As measured on the SSI, midtreatment remission rates were $33.3 \% \%$ for ABFT/HSA versus $37.5 \%$ for EUC/HAS, $\chi^{2}(1)=0.04, p=.84$, OR $=0.80$. Posttreatment remission rates were $69.2 \%$ for ABFT/HSA vs. $11.1 \%$ for EUC/HSA, $\chi^{2}(1)=7.25, p=.007$, OR $=17.99$. Follow-up remission rates were $78.6 \%$ for ABFT versus $50 \%$ for EUC, $\chi^{2}(1)=1.92, p=.17, \mathrm{OR}=3.67$.

Remission rates for depression. Among adolescents with HSA, those in ABFT were more likely to exhibit remission of depression than those in EUC. This difference was significant at posttreatment and follow-up. Midtreatment remission rates were $17.7 \%$ for ABFT/HSA versus $0 \%$ for EUC/HSA (Fisher's exact, $p=.26$ ), corresponding to an infinite odds ratio. Posttreatment remission rates were $47.1 \%$ for ABFT/HSA versus $9.1 \%$ for EUC/ HSA (Fisher's exact, $p=.049, \mathrm{OR}=8.99$ ). Follow-up remission

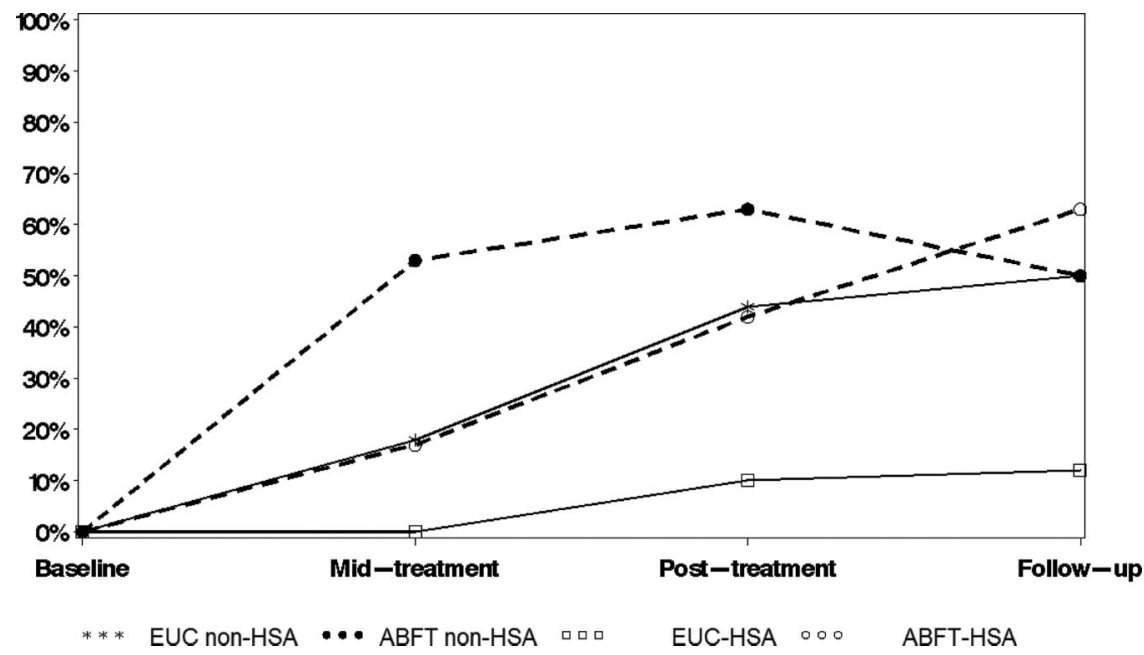

Figure 5. Proportion scoring in normative range on depression (BDI). 
rates were $64.7 \%$ for ABFT/HSA versus $12.5 \%$ for EUC/HSA (Fisher's exact, $p=.03$, OR $=12.83$ ).

Impact of attrition. ABFT patients attended a significantly greater number of sessions $(M=9.71, S D=5.26)$ than EUC patients $(M=2.87, S D=3.3 ; Z=-4.74, p<.001)$. Various missing data patterns were combined to two clinically meaningful patterns: completers, corresponding to patients with complete data, and noncompleters (see Hedeker \& Gibbons, 1997, and Barber, Barrett, Gallop, Rynn, \& Rickels, in press) Implementations of the pattern-mixture approach indicated that our findings were not biased due to completion. Additionally, we assessed whether number of sessions attended impacted our findings (22 cases came to 10 session or more, while six cases came to less than six sessions). Implementations of the pattern-mixture approach indicated that our findings were not biased due to sessions attended.

\section{Discussion}

In contrast to the existing treatment outcome literature, the presence of a sexual trauma history did not moderate the efficacy of ABFT when treating adolescents for elevated suicide ideation and depression. ABFT was more effective than EUC regardless of whether adolescents had a HSA. This finding was fairly consistent for suicide ideation and depression and when examined for both rates of change and remission. These findings are particularly important in light of several recent studies suggesting that depressed adolescents with a trauma history did not respond well to CBT (Barbe et al., 2004; Lewis et al., 2010), even in combination with medication (Asarnow et al., 2009). Given the low attendance rates of the EUC treatment, it is possible that the between group difference is a function of staying in therapy and not ABFT. However, when comparing effect sizes between ABFT in this study and CBT in the TADS study, we found ABFT performed better than CBT with patients that had a HSA. These findings are very promising. A comparison, in a randomized clinical trial, would be more conclusive.

At baseline, we found that a history of one or more suicide attempts differentiated those with a HSA and those without. This indicator has been associated with more severely disturbed patients, which may partially explain why these patients might be more complicated to treat (Oquendo et al., 2005). The fact that AFBT was successful with these patients makes further exploration with this treatment modality important. We also found that baseline rates of reported HSA in this sample were much higher $(46 \%)$ than in the referenced depression treatment studies: $8.9 \%$ in the TADS study (Lewis et al., 2010), 10\% in the Barbe et al. (2004) study. The higher prevalence of a HSA in the current sample may be a function of the inclusion criteria. A sample recruited for elevated suicide ideation is likely to have chronic family problems which may lead to, or result from, higher rates of sexual trauma histories. However, in the Treatment of Adolescent Suicide Attempts (TASA; Brent et al., 2009), only 17\% of patients reported a history of sexual abuse. Our higher rates of HSA may also be a function of more thorough screening procedures or the low income, urban nature of our sample population which is associated with more chronic stress and abuse (Conger et al., 2002).

Given the differential outcomes on comparisons of effect sizes, we can speculate as to why ABFT might perform better than CBT with this population. As Lewis et al. (2010) and Asarnow et al. (2009) suggest, CBT manuals in randomized trials are semistructured, thus perhaps limiting the flexibility of the therapist to focus on trauma. In contrast, the ABFT manual directs therapists to look for core family conflicts to serve as the content and focus of treatment. Although the trauma typically occurred in the past rather than acutely in all these studies, a trauma history might have clinical meaning for adolescents. In one ABFT case, the sexual trauma had been reported and prosecuted 3 years before treatment. However, the daughter felt the mother had never believed her about the assault, and harbored resentment about breaking up the mother's (perceived) positive and financially helpful relationship with the perpetrator. ABFT treatment focused on helping the daughter express these unspoken feelings and helped the mother understand the impact of her ambivalence and mixed messages. These discussions helped reduce family tension, the daughter's self-punishing guilt (which manifested in suicide behavior), and the mother's anger. The ABFT manual gave the therapist enough flexibility to use the clients' personal "hot topics" as therapeutic material to teach new interpersonal skills, even if the trauma was not recent and acute.

The different mechanisms of change in CBT and ABFT may also contribute to these findings. A core mechanism of CBT is to identify, evaluate, and modify inaccurate or unhelpful thoughts and beliefs to reduce distress and impairment in the adolescent (Creed, Reisweber, \& Beck, 2011). Changes in thinking and beliefs are the proposed mediator of change (Weersing, Rozenman, \& Gonzalez, 2009). Adolescents are coached to problem-solve real world concerns, but these efforts focus on how the adolescent might think and act differently in response to these situations, with support from parents when possible (Creed et al., 2011). However, as Barbe et al. (2004) points out, sexual abuse may be indicative of a chronically dysfunctional family environment. Learning, assimilating, and using better cognitive coping skills may be difficult in the face of constant environmental and psychological stress.

In contrast to CBT, ABFT aims at both individual and family level change. From an attachment perspective, without the expectancy of a safe and protective parenting environment, adolescents will not engage in the types of family conversations in which interpersonal problem solving and emotion regulation is learned (Adrian, Zeman, Erdley, Lisa, \& Sim, 2011; Sheeber, Hops, \& David, 2001). Therefore, we first help adolescent resuscitate their desire for parental love and protection. Then, we supportively explore parents' personal barriers to more effective caregiving and teach them more emotion-focused parenting skills (Gottman et al., 1996). We assist adolescent disclosure of past or current personal and interpersonal challenges. If parents respond with support and empathy, adolescents gain confidence in parents' availability. In this regard, not only do family members' interactional patterns change but their very conceptions of themselves and the other (i.e., cognitive schema) are thought to change as well. In theory, adolescents begin to perceive themselves as more worthwhile and their parents as more caring and available. As a consequence, adolescents more likely seek parental protection during moments of distress, thus reducing their risk for suicide. While we may or may not address a history of sexual abuse, we aim to repair the personal and interpersonal process that may have allowed abuse to occur. 
Although this study had a number of important findings, some limitations should be noted. The sample size was small, increasing the likelihood that outliers may affect results. However, analysis indicated there was no outlier effect. Further, based on the observed posttreatment remission rates for the SIQ-JR and the BDI, a sample size of 265 and 194 would be required to achieve $80 \%$ power based on observed effects, a very larger sample for this population. The sample was also mostly urban, African American females, which may limit generalizability. The definitions and methods used to identify HSAs were more detailed than other similar studies, but were not as thorough as possible.

Sexual trauma can devastate a child's sense of safety, security, and self-worth in ways that can last a lifetime (Mannarino \& Cohen, 2006). ABFT aims to improve communication, problem solving, trust, and safety. This may create a secure base within which adolescent feel more comfortable exploring troubling events like sexual abuse or its consequences. This treatment helps the adolescent receive acknowledgment, information, and even an apology about past traumas and negative family processes that may accelerate how adolescents remember, think about, and understand these events. As parents help the adolescent work through these difficult experiences, a new history of positive interactions develops; a corrective attachment experience. We hypothesize that these changes in the emotional and trustworthiness of family life would allow these troubled adolescents to be more receptive to CBT strategies which could further help modify negative memories and perceptions of self and other. The efficacy of ABFT alone, and/or in combination with CBT, for treating suicidal youth with an HSA warrants further investigation.

\section{References}

Adrian, M., Zeman, J., Erdley, C., Lisa, L., \& Sim, L. (2011). Emotional dysregulation and interpersonal difficulties as risk factors for nonsuicidal self-injury in adolescent girls. Journal of Abnormal Child Psychology, 39, 389-400. doi:10.1007/s10802-010-9465-3

Asarnow, J. R., Emslie, G., Clarke, G., Wagner, K. D., Spirito, A., Vitiello, B., \& Brent, D. (2009). Treatment of selective serotonin reuptake inhibitorresistant depression in adolescents: Predictors and moderators of treatment response. Journal of the American Academy of Child and Adolescent Psychiatry, 48, 330-339. doi:10.1176/appi.ajp.2010.09040552

Barbe, R., Bridge, J., Birmaher, B., Kolko, D., \& Brent, D. (2004). Lifetime history of sexual abuse, clinical presentation, and outcome in a clinical trial for adolescent depression. Journal of Clinical Psychiatry, 65, 77-83. doi:10.4088/JCP.v65n0113

Barber, J. P., Barrett, M. S., Gallop, R., Rynn, M., \& Rickels, K. (in press). Short-Term psychodynamic therapy vs. pharmacotherapy for major depressive disorder, Journal of Clinical Psychiatry.

Beautrais, A., Joyce, P., \& Mulder, R. (1996). Risk factors for serious suicide attempts among youth aged 13-24. Journal of the American Academy of Child and Adolescent Psychiatry, 35, 1174-1182. doi: 10.1097/00004583-199609000-00015

Beck, A. T., Kovacs, M., \& Weissman, A. (1979). Assessment of suicidal intention: The Scale for Suicide Ideation. Journal of Consulting and Clinical Psychology, 47, 343-352. doi:10.1037/0022-006X.47.2.343

Beck, A. T., Steer, R. A., \& Brown, G. K. (1996). Manual for Beck Depression Inventory-II (2nd ed.). San Antonio, TX: Psychological Corporation.

Belik, S., Cox, B. J., Stein, M. B., Asmundson, G. J. G., \& Sareen, J. (2007). Traumatic events and suicidal behavior: Results from a national mental health survey. Journal of Nervous and Mental Disease, 195, 342-342-349. doi:10.1097/01.nmd.0b013e318060a869
Brent, D. A., Greenhill, L. L., Compton, S., Emslie, G., Wells, K., Walkup, J. B., .. Turner, J. B. (2009). The Treatment of Adolescent Suicide Attempters study (TASA): Predictors of suicidal events in an open treatment trial. Journal of the American Academy of Child Adolescent Psychiatry, 48, 987-996. doi:10.1097/CHI.0b013e3181b5dbe4

Brent, D. A., Holder, D., Kolko, D., Birmaher, B., Baugher, M., Roth, C., ... Johnson, B. A. (1997). A clinical psychotherapy trial for adolescent depression comparing Cognitive, Family, and Supportive Therapy. Archives of General Psychiatry, 54, 877-885. doi:10.1001/ archpsyc.1997.01830210125017

Brown, G., Have, T., Henriques, G., Xie, S., Hollander, J., \& Beck, A. (2005). Cognitive therapy for the prevention of suicide attempts: A randomized controlled trial. Journal of the American Medical Association, 294, 563-570. doi:10.1001/jama.294.5.563

Centers for Disease Control and Prevention (CDC). (2005). Web-based Injury Statistics Query and Reporting System (WISQARS). Atlanta, GA: National Center for Injury Prevention and Control. Retrieved July 20, 2010 from www.cdc.gov/ ncipc/wisqars/default.htm

Conger, R. D., Wallace, L. E., Sun, Y., Simons, R. L., McLoyd, V. C., \& Brody, G. H. (2002). Economic pressure in African American families: A replication and extension of the family stress model. Developmental Psychology, 38, 179-193. doi:10.1037/0012-1649.38.2.179

Creed, T. A., Reisweber, J., \& Beck, A. T. (2011). Cognitive therapy for adolescents in school settings. New York, NY: Guilford Press.

David-Ferdon, C., \& Kaslow, N. J. (2008). Evidence-based psychosocial treatments for child and adolescent depression. Journal of Clinical Child and Adolescent Psychology, 37, 62-104. doi:10.1080/1537 4410701817865

Diamond, G. M., Diamond, G. S., Levy, S., Closs, C., Ladipo, T., \& Siqueland, L. (in press). Attachment-based family therapy for suicidal lesbian, gay and bisexual adolescents: A treatment development study and open trial with preliminary findings. Psychotherapy.

Diamond, G. S., \& Liddle, H. A. (1999). Transforming Negative ParentAdolescent Interactions: From impasse to dialogue. Family Process, 38, 5-26. doi:10.1111/j.1545-5300.1999.00005.x

Diamond, G. S., Reis, B. F., Diamond, G. M., Siqueland, L., \& Isaacs, L. (2002). Attachment-based family therapy for depressed adolescents: A treatment development study. Journal of the Academy of Child \& Adolescent Psychiatry, 41, 1190-1196. doi:10.1097/00004583200210000-00008

Diamond, G. S., Siqueland, L., \& Diamond, G. M. (2003). Attachmentbased family therapy: Programmatic treatment development. Clinical Child and Family Psychology Review, 6, 107-127. doi:10.1023/A: 1023782510786

Diamond, G. S., \& Wintersteen, M. (2007). Reasons for suicide questionnaire. Unpublished manuscript.

Diamond, G. S., Wintersteen, M. B., Brown, G. K., Diamond, G. M., Gallop, R., Shelef, K., \& Levy, S. A. (2010). Attachment-based family therapy for suicidal adolescents: A randomized controlled trial. Journal of the American Academy of Child and Adolescent Psychiatry, 49, 122-131. doi:10.1016/j.jaac.2009.11.002

Gelman, A., \& Hill, J. (2007). Data analysis using regression and multilevel/hierarchical models. New York, NY: Cambridge University Press. doi:10.2277/0521867061

Gottman, J. M., Katz, L. F., \& Hooven, C. (1996). Parental meta-emotion philosophy and the emotional life of families: Theoretical models and preliminary data. Journal of Family Psychology, 10, 243-268. doi 10.1037/0893-3200.10.3.243

Grothe, K. B., Dutton, G. R., Jones, G. N., Bodenlos, J., Ancona, M., \& Brantley, P. J. (2005). Validation of the Beck Depression Inventory-II in a Low-Income African American Sample of Medical Outpatients. Psychological Assessment, 17, 110-114. doi:10.1037/1040-3590.17.1.110 Hedeker, D., \& Gibbons, R. D. (1997). Application of random-effects 
pattern-mixture models for missing data in longitudinal studies. Psychological Methods, 2, 64-64-78. doi:10.1037/1082-989X.2.1.64

Joiner, T., \& Coyne, J. C. (1999). The interactional nature of depression: Advances in interpersonal approaches. Washington, DC: American Psychological Association. doi:10.1037/10311-000

Kazdin, A. E. (2001). Progression of therapy research and clinical application of treatment require better understanding of the change process. Clinical Psychology: Science \& Practice, 8, 143-151. doi:10.1093/ clipsy.8.2.143

Kraemer, H. C., Wilson, T., Fairburn, C. G., \& Agras, W. S. (2002). Mediators and moderators of treatment in randomized clinical trials. Archives of General Psychiatry, 59, 877-883. doi:10.1001/archpsyc .59 .10 .877

Lewis, C. C., Simons, A. D., Nguyen, L. J., Muakami, J. L., Reid, M. W., Silva, S. G., \& March, J. S. (2010). Impact of childhood trauma on treatment outcome in the Treatment for Adolescents with Depression Study (TADS). Journal of the American Academy of Child Adolescent Psychiatry, 49, 132-140. doi:10.1097/00004583-201002000-00007'

Mannarino, A. P., \& Cohen, J. A. (2006). Child sexual abuse. In R. T. Ammerman (Ed.), Comprehensive handbook of personality and psychopathology (Vol. 3, pp. 388-402). Hoboken, NJ: John Wiley \& Sons Inc.

Oquendo, M., Brent, D. A., Birmaher, B., Greenhill, L., Kolko, D., \& Stanley, B., (2005). Posttraumatic stress disorder comorbid with major depression: Factors mediating the association with suicidal behavior. The American Journal of Psychiatry, 162, 560-566. doi:10.1176/ appi.ajp.162.3.560
Promising Practices Network. (2011). Retrieved from www.promisingpractices.net

Reynolds, W. M. (1988). Suicidal Ideation Questionnaire. Professional Manual. Odessa, FL: Psychological Assessment Resources.

Reynolds, W. M., \& Mazza, J. (1999). Assessment of suicidal ideation in inner-city children and young adolescents: Reliability and validity of the Suicidal Ideation Questionnaire-JR. School Psychology Review, 28, 17-30.

Rosenbaum, P. R. (2002). Observational studies. New York, NY: Springer. Shaffer, D., Fisher, P., \& Lucas, C., (1997). NIMH DISC-IV: Diagnostic Interview Schedule for Children. Parent Informant (Interview about Child)_Epidemiologic Version. New York, NY: Columbia University.

Sheeber, L. B., Hops, H., \& Davis, B. (2001). Family process in adolescent depression. Clinical Child and Family Psychology Review, 4, 19-35. doi:10.1023/A:1009524626436

Ullman, S., \& Brecklin, L. (2002). Sexual assault history and suicidal behavior in a national sample of women. Suicide and Life-Threatening Behavior, 32, 117-130. doi:10.1521/suli.32.2.117.24398

Weersing, V. R., Rozenman, M., \& Gonzalez, A. (2009). Core components of therapy in youth: Do we know what to disseminate? Behavioral Modification, 33, 24-47. doi:10.1177/0145445508322629

Received April 12, 2011

Revision received February 29, 2012

Accepted March 5, 2012 